\title{
Perfil Antropométrico de Jugadores Profesionales de Voleibol Sudamericano
}

\author{
Anthropometric Profile of Professional Volleyball Sudamerican Players
} *Atilio Aldo Almagià Flores; "Fernando Rodríguez Rodríguez; "Fernando Omar Barrraza Gómez;
"Pablo José Lizana Arce; "Daniza Ivanovic Marincovich \& ${ }^{* * * *}$ Octavio Binvignat Gutiérrez

ALMAGIÀ, A. F.; RODRIGUEZ, R. F. J.; BARRAZA, G. F. O.; LIZANA, P. J.; IVANOVIC, D. \& BINVIGNAT, G. O. Perfil antropométrico de jugadores profesionales de voleibol sudamericano. Int. J. Morphol., 27(1):53-57, 2009.

RESUMEN: El voleibol es beneficiado por la aplicación de la antropometría, tanto por la evaluación de la composición corporal, que predice los rendimientos fisiológico y deportivo y define comportamientos mecánicos, para determinar la posición más eficiente dentro del campo de juego, de acuerdo a las características antropométricas. Se evaluaron las selecciones nacionales adultas de Colombia, Paraguay, Uruguay, Venezuela y la Selección Juvenil de Chile, utilizando el método de fraccionamiento corporal y el Somatotipo de Heath-Carter. El equipo Vicecampeón Sudamericano, Venezuela, fue el que tenía mayores porcentajes de masa muscular y bajos porcentajes de masa adiposa. El somatotipo de los equipos presentaba una mesomorfismo aumentado, seguido de un alto ectomorfismo, obteniendo la clasificación de individuos ecto-mesomórficos. La utilización de los datos obtenidos contribuirá a formar un modelo de jugador con una morfología estructuralmente ideal, capaz de afrontar competencias de alto nivel competitivo. La información obtenida ayuda a orientar el trabajo deportivo de manera certera y eficiente para la detección de talentos o entrenamiento de futuros voleibolistas profesionales.

PALABRAS CLAVE: Somatotipo; Composición corporal; Antropometría; Voleibol.

\section{INTRODUCCIÓN}

Uno de los factores más importantes y determinantes de un buen rendimiento en voleibol, es la estatura. En un estudio de Papadopoulou (Papadopoulou, 2001), muestra las características antropométricas de los mejores jugadores de voleibol del mundo, con una edad promedio de $26 \pm 3,6$ años, un promedio de $93,26 \pm 5,8 \mathrm{Kg}$ de peso y una estatura promedio de $199 \pm 7,2 \mathrm{~cm}$. La cantidad de grasa corporal es también un factor fundamental sobre el rendimiento deportivo; ejemplo de ello, es la selección de voleibol de Grecia cuyos integrantes presentaban un porcentaje de grasa de $12,59 \pm 4$ con el protocolo de Jackson \& Pollock, (Jackson \& Pollock, 1978), lo cual es bajo en comparación al de otros deportistas (Papadoupolou).

En voleibol, la altura de la malla se encuentra a 2,43 metros, por lo tanto aquellos jugadores más altos tienen que realizar esfuerzos en salto relativamente menores para poder superar la altura de la red. Los bloqueadores y remachadores ocupan alrededor de 7 a 15 minutos en un partido, realizando esfuerzos en saltos, por lo que la consecución de repetidos saltos explosivos son en conjunto con la talla claves en el éxito (Norton \& Olds, 2000).

En voleibolistas adolescentes se presenta una frecuente reducción de la cantidad de grasa corporal en sesiones de entrenamiento de 2 horas, con una frecuencia de 5 días por semana (Abreu \& Abreu, 2003). De esta manera, el voleibol es beneficiado por la aplicación de la antropometría, tanto por la evaluación de la composición corporal, que predice los rendimientos fisiológico y deportivo, como por las medidas sin procesar en fórmulas, como las longitudes y alturas, que pueden definir comportamientos mecánicos, para determinar la posición más eficiente dentro del campo de juego, de acuerdo a las características antropométricas.

\footnotetext{
* Laboratorio de Antropología Física y Anatomía Humana, Instituto de Biología, Fac. de Ciencias, Pontificia Universidad Católica de Valparaíso, Chile.

*** Instituto de Nutrición y Tecnología de los Alimentos, Universidad de Chile, Chile.

*** Caxias do Sul- RS- Brasil, Profesor consultor y asesor Laboratorio de Antropología Física y Anatomía Humana, Instituto de Biología, Facultad de Ciencias, Pontificia Universidad Católica de Valparaíso. Chile.
} 
En Chile, se desconoce el uso de la antropometría, para los propósitos anteriores y se hace necesario comparar y determinar la composición corporal y el somatotipo de los seleccionados chilenos v/s selecciones nacionales de otros países sudamericanos, para postular una caracterización de referencia en voleibolistas de elite.

\section{SUJETOS Y MÉTODO}

Los sujetos fueron voluntarios, no exigidos y previamente instruidos en lo qué consistirá su participación. Las evaluaciones se realizaron durante el Campeonato Panamericano de Voleibol disputado en Chile, en el año 2007, y en el Centro de Alto Rendimiento (CAR) durante el 2008.

Se evaluaron jugadores de las selecciones nacionales adultas de Colombia (6), Paraguay (7), Uruguay (13), Venezuela (10) y la Selección Juvenil de Chile (12). (Tabla I).

Tabla I. Medianas estadísticas de los datos básicos de los individuos evaluados.

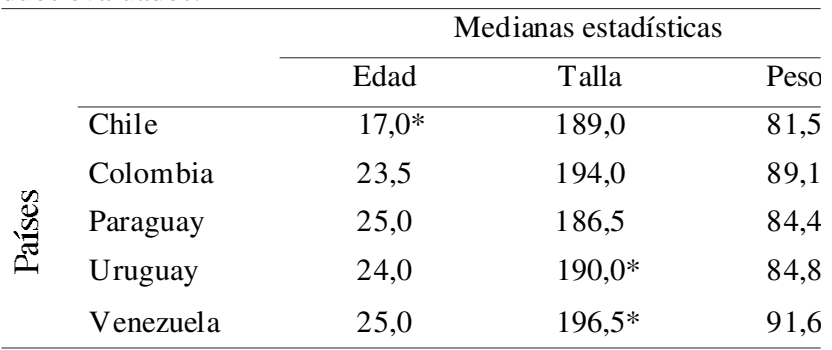

* Diferencias significativas entre los grupos evaluados según prueba de contraste de Kruskall-Wallis.

En este estudio hemos utilizado el método de fraccionamiento corporal de Dhebora Kerr (Kerr, 1988), primer estudio validado por el estudio de Bruselas de disección de cadáveres (Clarys et al., 1984) tanto para pesos estructurados y obtenidos como para las distintas masas corporales. Éste es un método pentacompartimental, que divide la morfoestructura en: masa adiposa (MA), masa muscular (MM), masa ósea (MO), masa residual y masa de la piel. Además, se utilizó el método antropométrico del somatotipo de Carter \& Heath (Carter \& Heath, 1990), método tricompartimental que divide la morfoestructura en masa muscular, masa ósea y masa grasa, entregando una idea de la forma corporal de los individuos evaluados. Para la recolección de las medidas y para el protocolo de medición se usó el kit antropométrico Rosscraft SRL validado por la Internacional Society for the Avancement in Kineanthropometric (ISAK), que incluye antropómetros óseos, huincha métrica y plicómetro o calibre para pliegues. Los datos se registraron en planillas de recolección manual y posteriormente, en pla- nillas Excel de computadora para su análisis básico y una prueba de contraste de Kruskall-Wallis como alternativa no paramétrica del método ANOVA, ya que no se utilizó una muestra escogida aleatoriamente, sino que voluntaria.

Las mediciones se realizaron bajo el protocolo de marcaje y medición de la ISAK, en condiciones normales de temperatura, a primera hora de la mañana y después del vaciado urinario, por evaluadores con licencia nivel II de la ISAK.

\section{RESULTADOS}

Dos diferencias notorias que fue posible apreciar entre los jugadores de los equipos sudamericanos estudiados correspondieron a la cantidad de MM y de MA.

La Tabla II muestra que el equipo chileno presenta un porcentaje de MA levemente superior a los otros equipos; lo cual trae como consecuencia un porcentaje MM menor que la de los otros equipos.

Los integrantes del equipo Vicecampeón Sudamericano, Venezuela, fueron los que poseían mayores porcentajes de MM y bajos porcentajes de MA, 18,92\% ubicándose según la clasificación de composición corporal ideal del laboratorio Biosystem de Argentina, en un rango “bueno”. Venezuela también incluía al jugador con menor porcentaje de MA con un 14,14\%, lo cual se considera "excelente". En Chile, al menos el $75 \%$ de los jugadores se encuentra en un estado de MA aceptable.

La Fig. 1, muestra que el equipo de Colombia presenta un porcentaje de masa adiposa con una mínima variabilidad, respecto de los otros cuatro equipos, lo cual lo hace un grupo más homogéneo. También indica que hay diferencias en las medianas entre todos los equipos.

La Fig. 2 muestra diferencias significativas en porcentaje MM entre las medianas de los equipos. Chile y $\mathrm{Pa}-$ raguay presentan menor variabilidad. El resto de los equipos se mantienen relativamente homogéneos, pero Venezuela con una mediana mayor que todos los demás equipos.

El somatotipo de los integrantes de los equipos presentaba un mesomorfismo aumentado, seguido de un alto ectomorfismo, obteniendo la clasificación de individuos ectomesomórficos, según Carter \& Heath.

En la Fig. 3, se muestra que los jugadores de los equipos de Paraguay y Uruguay poseían una forma corporal pre- 
Tabla II. Número, medias, desviaciones estándar, mínimos y máximos de los porcentajes de masa adiposa y masa muscular de los jugadores profesionales de voleibol sudamericano de los cinco equipos de evaluados.

\begin{tabular}{ccccccc}
\hline Variable & País & $\mathrm{n}$ & Media & DV & Mínimo & Máximo \\
\hline \multirow{3}{*}{ \% Masa adiposa } & Chile & 12 & 21,63 & 4,64 & 16,02 & 33,61 \\
& Colombia & 6 & 20,65 & 0,82 & 20,10 & 22,27 \\
& Paraguay & 7 & 20,24 & 2,78 & 17,00 & 25,20 \\
& Uruguay & 13 & 20,42 & 2,87 & 15,93 & 24,30 \\
& Venezuela & 10 & 18,92 & 3,31 & 14,14 & 25,30 \\
& & & & & & \\
\hline \multirow{2}{*}{ Masa muscular } & Chile & 12 & 49,09 & 6,49 & 30,00 & 54,56 \\
& Colombia & 6 & 51,24 & 3,73 & 46,60 & 56,76 \\
& Paraguay & 7 & 51,39 & 1,80 & 49,35 & 53,82 \\
& Uruguay & 13 & 53,00 & 3,49 & 48,36 & 60,27 \\
& Venezuela & 10 & 53,23 & 2,57 & 49,34 & 56,35 \\
\hline
\end{tabular}

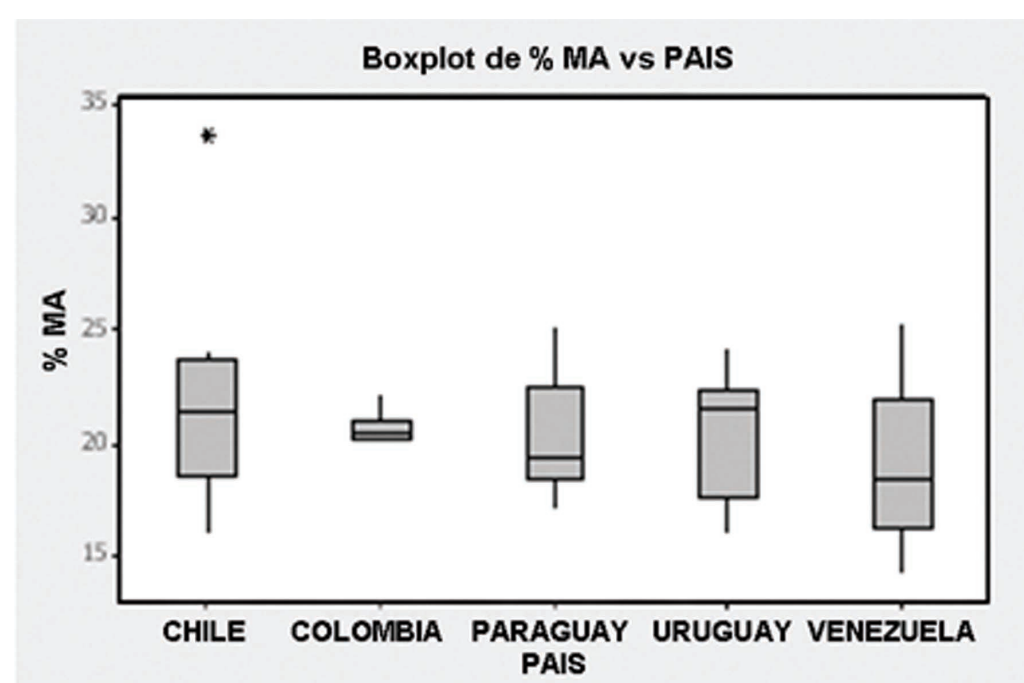

Fig. 1. Boxplot de las medianas y amplitudes de los porcentajes de MA de los cinco equipos evaluados.

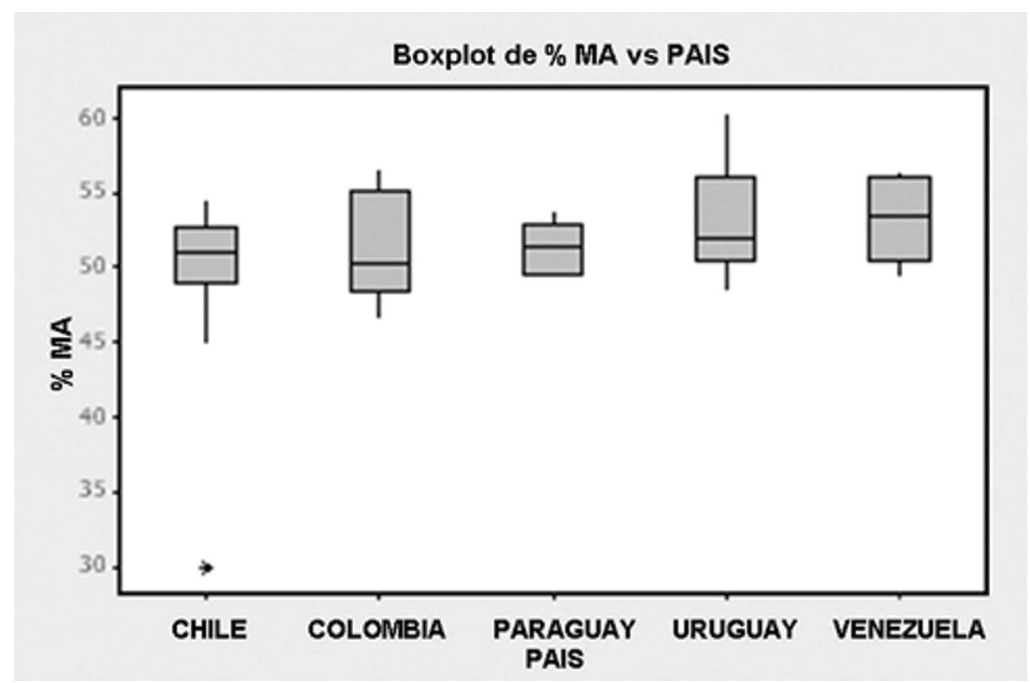

Fig. 2. Boxplot de las medianas y amplitudes de los porcentajes de MM de los cinco equipos evaluados. dominada por la MM mayor que los equipos de Venezuela, Colombia y Chile. A la vez que Paraguay y Uruguay presentaban un ectomorfismo más bajo, dando cuenta de una forma menos lineal con longitudes predominantemente menores que las presentadas por los jugadores de los equipos de Venezuela, Colombia y Chile. Además, cada uno de los componentes del somatotipo de los integrantes de los 5 equipos, se clasifican de la siguiente manera (Norton \& Olds, 1996): 1.- Baja adiposidad relativa, poca grasa subcutánea, contornos musculares y óseos visibles. 2.- Moderado y alto desarrollo músculo esquelético relativo, diámetros óseos grandes, mayor volumen muscular y articulaciones de grandes dimensiones. 3.- Linearidad relativa moderada, menos volumen por unidad de altura, más estirado.

En la Fig. 3. se presentan las diferencias establecidas en el mesomorfismo, siendo significativamente más alto en los jugadores de los equipos de Paraguay y Uruguay en comparación con los integrantes de los otros tres equipos. 


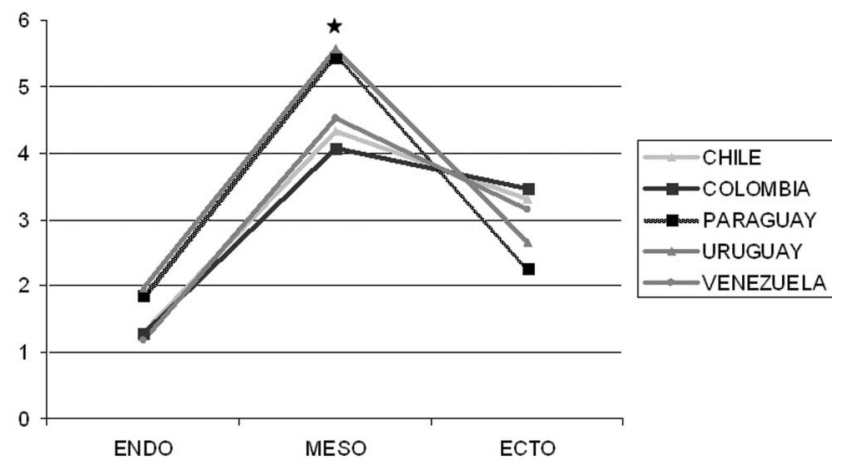

Fig. 3. Componentes del somatotipo: Endomorfismo (ENDO), Mesomorfismo (MESO) y Ectomorfismo (ECTO) de los cinco equipos evaluados.

\section{DISCUSIÓN}

Los resultados obtenidos permitieron comparar la morfoestructura de los jugadores de distintos equipos. El equipo de Venezuela se destacó por presentar los valores promedios más altos de MM y más bajos de MA.

De los integrantes de los cinco equipos evaluados durante el campeonato, es necesario destacar que los del equipo de Venezuela fueron los de mejor rendimiento, llegando hasta la semifinal y obteniendo el tercer lugar a nivel sudamericano. Un factor importante que influyó fue la altura de sus jugadores, quienes tienen un promedio de $196 \mathrm{~cm}$, siendo los más altos y significativamente distintos (Tabla I).

Existen otros factores que influyen sobre el rendimiento en Voleibol, como el tiempo dedicado a entrenar y los años de experiencia en el deporte. Una muestra de esto es que el equipo Chileno evaluado poseía una edad promedio de 17 años, significativamente menor que la del resto de los grupos (Tabla III), pero que aún se encontraba en edad madurativa para alcanzar mayor altura (Bellendier, 2001).
Además, los jugadores de este equipo presentaban poca experiencia y entrenamiento, obteniendo los valores más altos de MA y además los valores más bajos de MM por falta de desarrollo madurativo, estando a tiempo de mejorar su morfoestructura en base a una adecuada nutrición y entrenamientos exigentes, Chile correspondía a un equipo juvenil con proyecciones altamente promisorias, estando cercano a los parámetros estructurales de los equipos sudamericanos.

Los jugadores con ciertas características morfoestructurales, como mayor altura, mayor MM y menor MA rechazan más alto, poseen mayor potencia en sus miembros superiores y logran mayor fuerza en sus movimientos, lo cual mejora la eficiencia mecánica de este deporte, obteniendo un rendimiento notable frente a sus oponentes (Sheppard et al., 2008).

En el somatotipo de los integrantes de todos los equipos se pudo apreciar un predominio del mesomorfismo y secundariamente el ectomoerfismo, manteniéndose homogéneos entre los que integraban a equipos de Uruguay y $\mathrm{Pa}-$ raguay y entre los de Venezuela, Colombia y Chile, característica de los jugadores de alto nivel deportivo (Gualdi-Russo \& Zaccagni, 2001).

La utilización de los datos adquiridos contribuirá a formar un modelo de jugador con una morfología ideal para enfrentar competencias de alto nivel competitivo. La información obtenida ayuda a orientar el trabajo deportivo de manera certera y eficiente para detectar talentos o entrenamiento de futuros voleibolistas profesionales.

AGRADECIMIENTOS. A Esteban Vergara por su ayuda en la evaluación; a los entrenadores de los equipos de Uruguay, Paraguay, Colombia, Venezuela y Chile. A la Vicerrectoría de Investigación y Estudios Avanzados. Dirección de Investigación de la Pontificia Universidad Católica de Valparaíso, Chile, por el apoyo y financiamiento de la investigación.

ALMAGIÀ, A. F.; RODRIGUEZ, R. F. J.; BARRAZA, G. F. O.; LIZANA, P. J.; IVANOVIC, D. \& BINVIGNAT, G. O. Anthropometric profile of professional volleyball Sudamerican players. Int. J. Morphol., 26(1):53-57, 2009.

SUMMARY: The application of anthropometry provides many benefits to the sport of volleyball, such as in the evaluation of body composition, predicting physiological and sporting performance and defining mechanical behavior, from which the most efficient position in the court may be determined in terms of anthropometric characteristics. The national teams from Columbia, Paraguay, Uruguay and Venezuela were evaluated along with the Chilean youth team, using the method of body division and the Heath-Carter Somatotype. The South American runners-up, Venezuela, possess greater percentages of muscle mass and low percentages for adipose tissue mass. The somatotype of the teams presents an increased mesomorphy, followed by high ectomorphy, thus classifying the individuals as ectomesomorphs. The use of the data acquired allows the formation of a model of the morpho-structurally ideal player for high level competition. The information obtained guides physical preparation in an accurate and efficient way, leading to the detection of new talent or helping train future professional volleyball players.

KEY WORDS: Somatotype; Body composition; Anthropometry; Volleyball. 


\section{REFERENCIAS BIBLIOGRAFICAS}

Abreu, T. \& Abreu, E. Nutritional and anthropometric profile of adolescent volleyball athletes. Rev. Bras. Med. Esporte., 9(4):198-203, 2003.

Bellendier, J. El biotipo en el voleibol masculino. Revista digital de Educación Física y Deportes, Buenos Aires, 7(40), 2001. www.efdeportes.com/efd40/biotipo.htm.

Carter, J. E. L. \& Heath, B. H. Somatotyping - development and applications. Cambridge, Cambridge University Press, 1990.

Clarys, J. P.; Martin, A. \& Drinkwater, D. Gross tissue weights in the human body by cadaver dissection. Hum. Biol., 56(3):459-73, 1984.

Gualdi-Russo, E. \& Zaccagni, L. Somatotype, role and performance in elite volleyball players. J. Sports Med. Phys. Fitness, 41:256-62, 2001.

Jackson, A. S. \& Pollock, M. L. Generalized equations for predicting body density. Br. J. Nutr., 40:497-504, 1978.

Kerr, D. A. An anthropometric method for fractionation of skin, adipose, bone, muscle and residual masses in males and females age 6 to 77 years. M.Cs. Kinesiology Tesis, Simon Fraser University, British Columbia, 1988.

Norton, K. \& Olds, T. Antropometrica. Marrickville, Sidney, Southwood Press, 1996.

Norton, K. \& Olds, T. Sofwere LifeSize. Release 1.0. UNSW. Copirigth (c) by Human Kinetics, 2000.

Papadopoulou, S. D. Anthropometric characteristics of Greek top volleyball players. The effect of demographic and socioeconomic factors on the differentiation between competition levels. PhD Dissertation, Department of Sport Medicine, Faculty of Physical Education and Sport Science, Aristotle University of Thessaloniki, Greece, 2001.

Sheppard, J. M.; Cronin, J. B.; Gabbett, T. J.; McGuigan, M. R.; Etxebarria, N. \& Newton, R. U. Relative importance of strength, power, and anthropometric measures to jump performance of elite volleyball players. J. Strength. Cond. Res., 22(3):758-65, 2008.

\author{
Dirección para correspondencia: \\ Prof. Atilio Aldo Almagià Flores \\ Avda. Brasil 2950 \\ Casilla 4059 \\ Pontificia Universidad Católica de Valparaíso \\ Valparaíso \\ CHILE
}

Fax: 56-32-212746

Email: aalmagia@ucv.cl

Recibido : 27-09-2008

Aceptado: 28-10-2008 
